\title{
ARTIGOS
}

\section{INDICADORES DE SUSTENTABILIDADE \\ PARA UMA ORGANIZAÇÃO DO SEGMENTO \\ DA CONSTRUÇÃO CIVIL PESADA}

SUSTAINABILITY INDICATORS FOR AN ORGANIZATION OF THE HEAVY CIVIL CONSTRUCTION SEGMENT

\section{RAFAEL FEYH JAPPU, DR.|SENAC \\ MARIANA FRANCISCON | UNOESC}

\begin{abstract}
RESUMO
O presente trabalho apresenta um estudo sobre a estruturação de indicadores de desempenho para a medição da sustentabilidade de um a organização do segmento da construção civil pesada. O objetivo do estudo foi desenvolver uma estrutura de indicadores de sustentabilidade, que envolve-se as áreas de meio ambiente, segurança do trabalho, saúde ocupacional e gestão de talentos, a fim de demonstrar a maturidade da organização em tornar-se uma empresa referente em sustentabilidade no seu segmento. Os procedimentos metodológicos adotados foram preponderantemente da pesquisa bibliográfica e documental. Por meio deste estudo foi possível estruturar vinte e três novas métricas, com indicadores focados na sustentabilidade, parametrizados e implantados nas obras.
\end{abstract}

PALAVRAS CHAVE: Indicadores; Qualidade; Sustentabilidade; Planejamento estratégico; Construção Civil.

\begin{abstract}
This work presents a study on the structuring of performance indicators for measuring the sustainability of an organization in the heavy civil construction segment. The objective of the study was to develop a structure of sustainability indicators, which involves the areas of environment, occupational safety, occupational health and talent management, in order to demonstrate the organization's maturity in becoming a reference company in Sustainability in its segment. The methodological procedures adopted were preponderantly of bibliographical and documentary research. Through this study it was possible to structure twenty-three new metrics, with indicators focused on sustainability, parameterized and implanted in the works.
\end{abstract}

KEYWORDS: Indicators; Quality; Sustainability; Strategic Planning; Civil construction. 


\section{INTRODUÇÃO}

O presente trabalho apresenta um estudo sobre a estruturação de indicadores de desempenho para a medição da sustentabilidade em um a organização do segmento da construção civil pesada.

A existência de um indicador de desempenho em uma organização permite uma análise muito mais abrangente e profunda sobre a efetividade de uma gestão e de seus resultados do que uma simples constatação. Além de promover a cultura para a excelência, medir resultados sistematicamente e de forma estruturada, é de fundamental importância para uma organização, uma vez que possibilita a comparação com ciclos ou períodos passados. (FNQ, 2015a).

Em um ambiente de forte concorrência como se verifica atualmente, as empresas vencedoras são aquelas que percebem e implementam mudanças necessárias que possam continuar satisfazendo o consumidor, não apenas fazendo pequenas monitorações (FERNANDES, 2004).

A necessidade de medir o desempenho por meio de indicadores é crescente em todas as organizações, pois um indicador de desempenho é uma informação quantitativa e/ou qualitativa que expressa o desempenho dos processos em termos de eficiência, eficácia ou nível de satisfação. Os indicadores funcionam como ferramentas que conduzem ao comportamento desejado e devem dar aos indivíduos o direcionamento que precisam para atingir os objetivos estratégicos da organização (FNQ, 2015b).

É importante destacar que muitas empresas possuem em seus planejamentos estratégicos, diversos objetivos a serem alcançados com o desenvolver dos anos e das atividades realizadas, e quando bem estruturados os indicadores dentro de uma organização os mesmos, passam a dar índices confiáveis e traçam uma projeção do alcance desses objetivos.

Dessa forma, que este estudo abordará uma empresa de construção civil pesada, ao qual já possuía alguns padrões de medição de desempenho por indicadores, que decidiu implantar indicadores que se envolvessem também as áreas de meio ambiente, segurança do trabalho, saúde ocupacional e gestão de talentos, a fim de desenvolver e estruturar indicadores que possam demonstrar maturidade da organização em tornar-se uma empresa referente em sustentabilidade.

\section{FUNDAMENTAÇÃO TEÓRICA}

Nesse capítulo serão apresentados os principais conceitos teóricos referentes aos indicadores utilizados para o desenvolvimento desse trabalho e das pesquisas na empresa. Iniciando pelo tema sobre indicadores de desempenho e seus principais atributos, terminando com os indicadores de sustentabilidade.

\subsection{Indicadores de desempenho}

Como definição um indicador é uma ferramenta que permite a obtenção de informações sobre uma dada realidade, tendo como característica principal um poder de sintetizar diversas informações, retendo apenas o significado essencial dos aspectos que estão sendo analisados (CAMPOS e MELO, 2008).

Indicadores são representativos qualificáveis, de processo, características de produtos e outras atividades. $\mathrm{O}$ objetivo é permitir aos gestores acompanhar os resultados obtidos pela organização, com propósitos de adotar medidas corretivas que se façam necessárias (LUCINDA, 2010).

Os indicadores de negócio destinam-se a avaliar a organização como uma entidade fornecedora de produto e prestadora de serviço, a seus clientes potenciais, por meio de mensuração de parâmetros estratégicos, principalmente em seus processos de interação com o ambiente externo. Esses indicadores denominam-se também indicadores de desempenho, que destinam-se basicamente à parte permanente análise por paetê do corpo gerencial da empresa (TAKASHINA, 2002).

De acordo com Campos e Melo (2008) dentro desses contextos, pode-se disser que os indicadores são ferramentas utilizadas pelas organizações para monitorar determinados processos quanto ao alcance de uma meta ou padrão mínimo de desempenho estabelecido.

Segundo Lucinda (2010), os indicadores podem ser classificados em indicadores de qualidade e também de não qualidade, onde é medido o total de itens produzidos corretamente dividido pelo total de itens, e o itens defeituosos divididos pelo total de itens consecutivamente. Os indicadores devem ser capazes de permitir aos gestores uma interpretação da realidade (LUCINDA, 2010).

Os indicadores da qualidade ainda podem ser divididos em corporativos e setoriais, ou mesmo estratégicos e operacionais (LUSTOSA, 2008).

No nível operacional temos os indicadores de processo, que devem representar todos os processos existentes na empresa e que sejam importantes na dinâmica de criação de um valor para o consumidor. Dos vários grupos de indicadores de níveis operacionais surgem os de necessidade tática (FERNANDES, 2004).

Já os indicadores estratégicos estão relacionados à gestão estratégica do sistema organizacional, e os indicadores operacionais fornecem informações sobre o 
desempenho dos processos (LUSTOSA, 2008). De acordo com Fernandes (2004), os indicadores estratégicos estão no último nível e representam a posição e o posicionamento da empresa.

A separação vertical dos indicadores ocorre pela própria necessidade diferenciada da informação para o gerenciamento, nos três patamares básicos em que se divide uma empresa, estratégico, tático e operacional.

Para Campos e Melo (2008), outro fator relevante é a finalidade dos indicadores. Eles servem para medir o grau de sucesso da implantação de uma estratégia em relação ao alcance dos objetivos estabelecidos. Entretanto, é fundamental que seja observado o fato de que um indicador muito complexo ou de difícil mensuração não é adequado, pois o custo nele empregado para sua obtenção, pode inviabilizar sua operacionalização.

\subsection{Principais atributos dos indicadores}

A tarefa básica de um indicador é expressar, da forma mais básica, e mais simples possível, uma determinada situação que se deseja avaliar. O resultado do indicador é uma fotografia do dado no momento, e demonstra sobre uma base de medidas o que está sendo feito no momento (FERNANDES, 2004).

Segundo Lustosa (2008), o principal objetivo dos indicadores são explicitar as necessidades e expectativas dos clientes, viabilizar a aplicação de metas do negócio, suporte a análises críticas dos resultados dos negócios, às tomadas de decisão e ao controle e planejamento, bem como contribuição para a melhoria de processos e produtos da organização. Para o autor os principais atributos dos indicadores são os seguintes:

Adaptabilidade: capacidade do indicador em dar resposta às mudanças de comportamento e exigência dos clientes, podendo ser desnecessários a qualquer momento, e portanto tende a ser substituídos;

- Representatividade: o indicador deve captar as etapas mis importantes e críticas dos processos;

- Simplicidade: o indicador deve ser fácil de aplicar e ser entendido;

- Rastreabilidade: facilidade de identificar a origem dos dados, seu registro e manutenção;

- Disponibilidade: facilidade de acesso e disponível a tempo;

- Economia: não se deve gastar muito tempo procurando dados, e o custo de obtenção dos mesmos devem ser menor que o benefício de obtê-los;

- Praticidade: 0 indicador deve funcionar na prática para tomada de decisões.
Para Fernades (2004) os indicadores precisam externar credibilidade e, para tanto, devem ser gerados de forma criteriosa, assegurando a disponibilidade dos dados e resultados mais relevantes no menor tempo e custo possível. Bem como precisa atender os critérios e atributos, destacando-se entre eles a seletividade, simplicidade e clareza, abrangência, rastreabilidade e acessibilidade, comparabilidade, estabilidade e rapidez de disponibilidade, bem como baixo custo de obtenção.

De acordo com Lucinda (2010), existem diferentes tipos de indicadores, dentre eles podemos citar:

- Indicadores Estratégico: aqueles que indicam o andamento da organização em direção a consecução de sua visão de futuro;

- Indicadores de produtividade: medem a relação de insumos do processo ou atividades e suas saídas (produtos). Medem a eficiência do processo e atividades.

- Indicador de Capacitação: medidas que expressa informações sobre a estimativa da produção, capacidade instalada e número de empregados.

- Indicadores de Efetividade: indicadores de impacto, aos quais medem os impactos do processo sobre a clientela.

- Indicadores de qualidade: Medem as medidas de satisfação ou insatisfação dos clientes. Resultados dos processos e de sua eficácia.

Considerando os atributos e tipos de indicadores, que a seguir apresentaremos, além dos já citados, a perspectiva dos indicadores de sustentabilidade.

\subsection{Indicadores de sustentabilidade}

Para Bellen (2006) o processo de desenvolvimento de indicadores de sustentabilidade deve contribuir para uma melhor compreensão do que seja desenvolvimento sustentável.

Os indicadores de sustentabilidade fornecem informações sobre o desempenho econômicos, ambientais e sociais da organização, relacionados aos seus aspectos materiais, que são aqueles que refletem impactos econômicos, ambientais e sociais significativos da organização ou influenciam substancialmente as avaliações e decisões das partes interessadas no negócio da organização (GRI, 2015).

A dimensão econômica da sustentabilidade trata dos impactos econômicos na organização e sobre as suas partes interessadas, assim como nos sistemas econômicos em nível local, nacional e global. A dimensão econômica ilustra o fluxo de capital entre as diferentes partes interessadas e os principais impactos econômicos da organização sobre a sociedade como um todo, levando em 
consideração seu desempenho econômico, sua presença no mercado, suas práticas de compras, etc. (GRI, 2015).

A dimensão ambiental da sustentabilidade refere-se aos impactos da organização sobre ecossistemas, incluindo aspectos bióticos e abióticos (GRI, 2015). Os Indicadores de desempenho ambiental visam demonstrar práticas organizacionais no sentido de minimizar os impactos ao meio ambiente decorrente de suas atividades. Esses indicadores referem-se ao uso de recursos naturais demonstrados, iniciativas de gerenciamento ambiental, os impactos significativos relacionados ao setor $\mathrm{e}$ a atividade e as respectivas ações de minimização (CAMPOS e MELO, 2008).

Conforme as diretrizes do GRI (2015) a dimensão social da sustentabilidade diz respeito aos impactos da organização sobre os sistemas sociais em que ela atua, nela abordam-se as práticas trabalhistas e direitos humanos, sociedade como um todo, e também a responsabilidade pelo produto entregue. Um dos aspectos relacionados com a dimensão social é treinamento e a educação, aonde consta o número de horas de treinamento realizado pelos colaboradores. Também é relevante citar a saúde e a segurança do trabalho, como um aspecto da dimensão social, aonde constam tipo de taxa de lesão, doenças ocupacionais, dias perdidos, colaboradores com alto risco, etc. (GRI, 2015).

\section{METODOLOGIA}

A caracterização metodológica desta pesquisa, segundo Vergara (2008) e Gil (2010), possui mais similaridades com pesquisa qualitativa; quanto aos objetivos, situa-se em três categorias: exploratória, descritiva e explicativa, os tipos de pesquisa, segundo os autores, não são mutuamente exclusivos, o que permite classificá-lo nestes três tipos.

Em relação aos procedimentos adotados para a coleta de dados, esta pesquisa foi composta preponderantemente da pesquisa bibliográfica e documental, pela qual foram levantados dados primários já existentes na organização, bem como todos os índices já apresentados pelas obras e empreendimentos em relatórios apresentados aos clientes.

\section{APLICAÇÃO E RESULTADOS}

A empresa analisada, e escolhida para o desenvolvimento desse projeto possui sede em Concórdia - SC e atua no ramo da Construção Civil Pesada, com foco na execução de obras e terraplanagem em empreendimentos de geração de energia hidrelétrica, solar e eólica, obras de artes especiais, edificações residenciais, comerciais e industriais.
Desde sua fundação, ela vem executando serviços em empreendimentos importantes no setor elétrico brasileiro, construções de pontes, realocação de estradas vicinais e núcleos comunitários. A partir de 2004 atua em empreendimentos de porte no setor de geração de energia elétrica através do uso de fontes renováveis, especializando-se na construção de PCHs, UHEs e Complexos Eólicos no Brasil.

Vem contribuindo para o desenvolvimento sustentável do país, obtendo destaque no mercado em que atua, prestando serviços com qualidade e respeito ao meio ambiente, criando parcerias com clientes e fornecedores. Em 2011 obteve a certificação de seu Sistema de Gestão da Qualidade, baseado nos requisitos da NBR ISO 9001:2008.

Buscando a melhoria contínua, a empresa desenvolveu um sistema de gestão integrada, denominados Sistema de Sustentabilidade $\left(S^{3}\right)$, nele atuam as áreas de qualidade, meio ambiente, saúde ocupacional, segurança do trabalho e também gestão de talentos.

Dentro da gestão estratégica da empresa, foram traçados alguns objetivos a serem alcançados pela gestão até o ano de 2020. O objetivo estratégico que corresponde à área da sustentabilidade é: "Tornar-se empresa referência em Ações de Sustentabilidade".

Através desse objetivo estratégico, foi composto um plano de ação para desenvolver essa competência antes da estimada data. Uma das formas encontradas foi através de acompanhamento de indicadores hoje relacionados às áreas da sustentabilidade da organização.

Na sequência foram realizadas reuniões com os responsáveis de cada área da sustentabilidade, e definidos prazos referente aos estudos e as definições de quais indicadores seriam utilizados.

Assim que definidos os indicadores, foram desenvolvidos memórias de cálculos, para que os números e índices entregues ao sistema de gestão mantivessem o mesmo padrão e a garantia dos dados informados.

Os mesmos foram encaminhados aos seus responsáveis nas obras e empreendimentos, para tabulação de dados e preenchimentos dos indicadores, ao qual devem ser encaminhado ao sistema de gestão mensalmente para compilação e análise crítica dos dados para a direção.

Foram então desenvolvidos e aprimorados indicadores para o início do ano de 2016, os mesmos veem sendo compilados em base de dados, e assim que encerrado o ciclo anual, as metas hoje definidas passarão por uma análise crítica podendo sofrer alterações (Ver Quadro 01 Indicadores de sustentabilidade).

Conforme apresenta o Quadro 01, após a coleta de dados de todos os indicadores referente ao ano 2016, os 
mesmos passaram por análise crítica, junto à diretoria da empresa, sendo assim definidos quais indicadores serão importantes para alcançar as premissas do planejamento estratégico da empresa rumo a sustentabilidade de seu negócio.

Em segundo momento, os indicadores serão analisados criticamente e levados para aprovação e homologação pela alta direção, os mesmos vão passar a ser uma informação documentada do Sistema de Gestão da Qualidade - SGQ.

Assim que inserido no SGQ da empresa, o mesmo será repassado para os chamados designados de sustentabilidade, pessoas aos quais são responsáveis pelo sistema de sustentabilidade de cada obra.

Cada obra, obrigatoriamente deverá preencher todos os indicadores, com dados precisos referentes ao mês anterior e encaminhar até o quinto dia útil para o sistema de gestão da qualidade corporativo.

Recebido os dados, deverá ser feita uma tabulação, validados juntos com as áreas responsáveis pela sustentabilidade e apresentado em reunião de coordenação mensal, aonde participam alta direção, engenheiros residentes e coordenadores corporativos.

\section{CONSIDERAÇÕES FINAIS}

Ao acompanharmos indicadores referentes às áreas que compõem o Sistema de Sustentabilidade, encontra-se uma facilidade em identificar e compreender quais são os pontos positivos e quais pontos ainda que necessitem de um acompanhamento, estudo e também ações a serem tomadas para que se possa alcançar as metas e objetivos. Através da pesquisa realizada e da formulação dos indicadores, foi possível verificar a importância de cada assunto tratado e acompanhar os dados concretos a fim de parametrizar ações, intensificar as tomadas de decisões e refletir sobre os pontos fortes da organização.

Os indicadores de sustentabilidade possibilitam que a organização faça a gestão de seus processos com bases em resultados reais com credibilidade e demonstrando o grau de evolução das práticas desenvolvidas no processo. Estruturar metas e controles visando o planejamento estratégico da organização, analisando-os criticamente junto à direção, permite que seja feita uma tomada de decisão e aplicados melhorias ao sistema de sustentabilidade, com vistas a maturidade da organização no segmento.

Foi possível concluir com o estudo, considerando as métricas e os controles já existentes na organização e com objetivo de implantar novas métricas com indicadores de sustentabilidade estruturados, parametrizados e implantados nas obras foi alcançado.

A continuação desse projeto se dará até o término de 2016, aonde será possível analisar criticamente junto ao compilado de indicadores de sustentabilidade, os quais são indispensáveis para a organização e quais sofrerão alterações com relação às metas estipuladas. Na sequência será verificado como o conjunto de todos os indicadores, juntamente com sua área de atuação, demonstram a maturidade da organização em tornar-se uma empresa de referência em sustentabilidade, contribuindo para alcançar o planejamento estratégico para 2020.

\section{REFERÊNCIAS}

BELLEN, Hans Michael Van. Indicadores de Sustentabilidade: Uma Análise Corporativa. Rio de Janeiro: Editora FGV, 2006.

Campos, L. M. S.; Melo, D. A. Indicadores de desempenho dos Sistemas de Gestão Ambiental (SGA): uma pesquisa teórica. Produção, v. 18, n. 3, p. 540555, 2008. Disponível em: <http://www.prod.org.br/ files/v18n3/v18n3a09.pdf>. Acesso em: 10/11/2016.

FERNANDES, Djair Roberto. Uma combinação sobre a construção de indicadores e sua importância para a gestão empresarial. Rev. FAE, Curitiba, v.7, n.1, p.1-18, jan./jun. 2004. Disponível em: <http://www.logfacilba.com.br/media/FAEIndicadores.pdf $>$. Acesso em: 18/10/2016.

LIRA, Waleska Silveira; CÂNDIDO, Gesinaldo Ataíde. Análise dos modelos de indicadores no contexto do desenvolvimento sustentável. Perspectivas Contemporâneas. Campo Mourão, v. 3, n. 1, p. 31-45, jan./jul. 2008. Disponível em: <http://revista.grupointegrado.br/revista/index.php/perspectivascontemporaneas/article/view/436/208>. Acesso em: 14/10/2016.

FUNDAÇÃO NACIONAL DA QUALIDADE - FNQ. Sistema de Indicadores. São Paulo, SP, 2014a. Disponível em: $<$ http://www.fnq.org.br/informe-se/publicacoes/e-books>. Acesso em: 10/10/2016.

GIL, Antonio Carlos. Como elaborar projetos de pesquisa. 5. ed. São Paulo, SP: Atlas, 2010.

GLOBAL REPORTING INITIATIVE - GRI. Diretrizes para relato de sustentabilidade. 2. Ed. 2015. Disponível 
em: $\quad<$ https://www.globalreporting.org/resourcelibrary/Brazilian-Portuguese-G4-Part-One.pdf>. Acesso em: 10/11/2016.

LUCINDA. Marco Antônico. Qualidade: fundamentos e práticas para graduação. Rio de Janeiro: Brasport, 2010

LUSTOSA, Leonardo. Planejamento e Controle da Produção. Rio de Janeiro: Elsevier, 2008.

TAKASHINA, Newton Tadachi \& FLORES, Mario Cesar Xavier. Indicadores da Qualidade e do Desempenho. Rio de Janeiro: Qualitymark. 2002.

VERGARA, S. C. Projetos e relatórios de pesquisa em administração. 13. ed. São Paulo, SP: Atlas, 2008.

\section{AUTORES}

ORCID: https://orcid.org/0000-0002-5477-2415

RAFAEL FEYH JAPPUR, DR. | Serviço Nacional de Aprendizagem Comercial | SENAC | Florianópolis, SC. Brasil | Correspondência para: R. Silva Jardim, 360 - Centro, Florianópolis - SC | E-maill: rjappur@gmail.com

MARIANA FRANCISCON. | Universidade do Oeste de Santa Catarina | Gestão da Qualidade | Videira, SC. Brasil | Correspondência para: Rua Paese, 198-Bairro Universitário - Videira - SC - CEP 89560-000 | E-maill: franciscon.mariana@gmail.com.br

\section{COMO CITAR ESTE ARTIGO}

JAPPUR, Rafael Feyh; FRANCISCON, Mariana. Indicadores de Sustentabilidade para uma Organização do Segmento da Construção Civil Pesada. MIX Sustentável, [S.I.], v. 4, n. 3, p. 97-102, out-mar. 2018. ISSN 24473073. Disponível em: <http://www. nexos.ufsc.br/index.php/mixsustentavel>. Acesso em: dia mês. ano. doi:https://doi.org/10.29183/2447-3073. MIX2018.v4.n3.97-102.

DATA DE ENVI0: 09/08/2017

DATA DE ACEITE: 03/08/2018 